\title{
INTORNO
}

\section{AD UNA CLASSE DI INTEGRALI ESPRIMIBILI CON SOLI LOGARITMI.}

\author{
(Nota di Carlo Marta Pruma, dottore aggregato in Matematica
} nella $R$. Università di Genova.)

$\mathrm{N}$

ella presente Nota io mi propongo di estendere agli integrali della forma

$$
\int \frac{f d x}{\theta^{\frac{1}{n+n}}}
$$

[dove $m$ è un numero primo, $n$ un intero positivo qualunque ed $f$ e $\theta$ rappresentano due polinomi interi rispetto alla $x$, pel secondo dei quali posto

$$
\theta=\prod_{b=0}^{b=g}\left(x-a_{b}\right)^{c}
$$

sieno tutti i $\left.c_{b}<m^{4}\right]$ il teorema che dimostrai nella mia Nota che ha per titolo: Proprietà di una classe di integrali di irrazionali algebrici possibili con soli logaritmi, pel caso di $n=1$. (Vedasi Tortourn, Annali di Matematica pura ed applicata, vol. IV, pag. 4.)

1. Rappresentiamo con \& una radice primitiva dell' equazione

$$
x^{n^{n}}-1=0
$$

e con $M_{K}$ funzioni intere della $x$, posto

$$
X_{\alpha, p}=\sum_{K=0}^{K=m^{n}-1}\left(\alpha^{\alpha+m^{n-1} p} \theta^{\frac{1}{m^{n}}}\right)^{K} M_{K}
$$

se l' integrale (1) è esprimibile, con soli logaritmi, come è noto, devesi avere

$$
\int \frac{f d x}{6^{\frac{1}{m m}}}=A \sum_{q=0}^{q=m^{n-1}-1} \sum_{p=0}^{p=m-1} \log X_{q, p}^{\alpha^{q+3 m^{n-1} p}}
$$


dove $A$ è una costante; e sappiamo pure, che se $X_{\gamma, x}$ è nullo per un valore $h_{0}$ della $x$, esiste sempre una potenza $\left(x-h_{0}\right)^{\beta_{\varphi, x}}$ di $x-h_{0}$ per la quale il rapporto

$$
\frac{X_{p, \chi}}{\left(x-h_{0}\right)^{\beta_{\varphi, x}}}
$$

conserva un valore finito e diverso da zero per $x=h_{0}$, e di piu che $\beta_{\varphi, \chi}$ è un'intero se $h_{0}$ non è eguale ad alcuno degli $a_{b}$; e nel caso contrario, $\beta_{\varphi, \chi}$ può anche essere frazionario, ma allora il denominatore della frazione rappresentata da $\beta_{\varphi, x}$ è divisore di $m^{n}$ (vedasi la mia Nota precitata, pag. 7); per cui posto

$$
Y_{\varphi, x}=X_{\varphi, x}^{m^{n}}, \mathrm{e}, m^{n} \beta_{\varphi, x}=\gamma_{\varphi, x}
$$

si vede che $Y_{\varphi, \chi}$ è della stessa forma (4) di $X_{\varphi, \chi}$, e $\gamma_{\varphi, \chi}$ è un intero; inoltre evidentemente si ha

$$
\frac{X_{\varphi, \chi}^{m^{n}}}{\left(x-h_{0}\right)^{m^{n} \beta_{\phi, \chi}}}=\frac{Y_{\varphi, \chi}}{\left(x-h_{0}\right)^{\eta_{\varphi, \chi}}}=Z_{\varphi, \chi}
$$

dove $Z_{\psi, x}$ rappresenta una funzione di $x$ nè nulla nè infinita per $x=h_{0}$.

Dalla (5) poi si deduce

$\int \frac{f d x}{\theta^{\frac{1}{m^{n}}}}=\frac{A}{m^{n}} \sum_{q=0}^{q=m^{n-1}-1} \sum_{p=0}^{p=m-1} \log \left(X_{q, p}^{m w}\right)^{\alpha^{q+m^{n-1} p}}=A_{1}^{q=m^{n-1}-1} \sum_{q=0}^{p=\sum_{p=0}} \sum_{p=0}^{1} \log Y_{q, p}^{x^{q+m^{n-1} p}}$,

essendo $\frac{A}{m^{2}}=A_{1}$ costante. E chiaro che se nella (7) scriviamo $X_{\phi, \chi}$ al luogo di $Y_{p, \%}$, che hanno la stessa forma (4), ed $A$ al luogo di $A_{1}$ che sono costanti, otteniamo un' espressione della forma (5), in cui le $X_{p, \chi}$ avendo la forma data dalla (4), possiamo supporre che se una di esse si annullasse per $x=h_{0}$, esisterebbe sempre una potenza intera $\left(x-h_{0}\right)^{y_{p}, x}$ di $x-h_{0}$ per la quale i duo rapporti

$$
\frac{X_{\varphi, x}}{\left(x-h_{0}\right)^{\gamma_{\varphi, \psi}}} \text { e } \frac{\left(x-h_{0}\right)^{\gamma_{\varphi, x}}}{X_{\varphi, x}^{\prime}}
$$

resterebbero finiti per $x=h_{0}$.

2. Poniamo quindi

$$
X_{\varphi, \chi}=\left(x-h_{0}\right)^{\gamma_{\xi}, \chi} Z_{\rho, \chi}
$$

dove $Z_{p, \chi}$ si conserva finita e differente da zero per $x=h_{0}$. Dalla differenzia- 
zione della (5) tenuto conto della (8) si ottiene

$$
f-A 9^{\frac{1}{m^{n}}} \sum_{q=0}^{q=n^{n-1}-1} \sum_{p=0}^{p=m-1} \alpha^{q+m^{k-1} p} \frac{Z_{q, p}^{\prime}}{Z_{q, p}}=\frac{A 9^{\frac{1}{m^{n}}}}{x-h_{0}} \sum_{q=0} \sum_{p=0}^{p=m^{n-1}-1} \gamma_{q, p^{2}} a^{q+m^{n-1} p},
$$

ma, per $x=h_{0}$, il primo membro dell' eguaglianza precedente non è infinito, mentre il secondo lo diverrebbe [ciò che risulta dal fatto che, anche ammesso $\theta=0$ per $x=h_{0}$, si atrebbe $\theta=\left(x-h_{0}\right)^{n} \theta_{1}, \theta_{1}$ essendo un polinomio intero diverso da zero, per $x=h_{0}$, e $\left.n<m^{\prime \prime}\right]$ quando non si avesse

$$
\left.\begin{array}{l}
q=m^{n-1}-1 \\
\sum_{q=0}^{n} \sum_{p=0}^{p=m-1} \gamma_{q, p} a^{q+m t^{n-1} p^{n}}=\sum_{q=0}^{q=m^{n-1}-1} a^{q}\left\{\gamma_{q, m-1} a^{(m-1) m m^{n-1}}+\right. \\
\left.+\gamma_{q, m-2} e^{(m-2) m^{n-1}}+\gamma_{q, m-3} a^{(n-3) m^{n-1}}+\cdots+\gamma_{q, 0}\right\}=0,
\end{array}\right\}
$$

per oui questa relazione deve essere verificata dagli interi $\gamma_{\varphi, \%}$ e da $\alpha$.

L' equazione

$$
x^{(m-1) m m^{n-1}}+x^{(m-2) m m^{n-1}}+x^{(m-3) m m^{n-1}}+\cdots+1=0
$$

è irriducibile, e perchè $\alpha$ è radice primitiva della (3), deve pure essere radice della (10) che ammette tutte o sole lo radjci primitive della suddetta equazione (3), quindi dowremo avere

$$
\alpha^{(m-1) m^{n-1}}+\alpha^{(m-2) m^{n-1}}+\alpha^{(m-3) m^{n-1}}+\cdots+1=0
$$

e moltiplicandone $\mathrm{i}$ due membri per

si ottiene

$$
\sum_{q=0}^{q=m^{n-1}-1} \gamma_{q, m-1} \alpha^{q}
$$

$$
\sum_{q=0}^{q=m^{n-1}-1} \alpha^{q}\left\{\gamma_{q, m-1} a^{(m-1) m^{n-1}}+\gamma_{q, m-1} \alpha^{(m n-2) m^{n-1}}+\gamma_{y, m-1} \alpha^{(m-3) m^{n-1}}+\cdots+\gamma_{q, m-1}\right\}=0
$$

risultato che, sottratto membro a membro dalla (9) ci fornisce

$$
\begin{gathered}
\sum_{q=0}^{q=m^{n-1}-1} a^{q}\left(\gamma_{q, m i-2}-\gamma_{q, m-1}\right) a^{(m-2) m^{n-1}}+ \\
\left.+\left(\gamma_{q, m-3}-\gamma_{q, m-1}\right) \alpha^{(m-3) m m^{n-1}}+\cdots+\gamma_{q, n}-\gamma_{q, m-1}\right\}=0 .
\end{gathered}
$$

Ora la (12) è soddisfatta da $\alpha$ radice di un' equazione irriducibile (10) di grado $(m-1) m^{n-1}$, e i coefficienti delle diverse potenze di a nella (12) sono interi, 
ed il grado della potenza più elevata di $\alpha$ in quest' ultima equazione non supera $(m-2) m^{n-1}+m^{n-1}-1=(m-1) m^{n-1}-1$, quindi è inferiore almeno di un' unità al grado della $(10)$, il che ci fornisce la serie seguente di eguaglianze

$$
\gamma_{\varphi, m-1}=\gamma_{\varphi, m-2}=\gamma_{\varphi, m-3}=\cdots=\gamma_{\varphi, 1}=\gamma_{\varphi, 0}
$$

0 ciò che torna lo stesso ci dà per risultato che gli $m$ esponenti $\gamma_{\varphi, \chi}$ che hanno eguale il primo indice o sono eguali tra loro. Ripetendo gli stessi calcoli e gli stessi ragionamenti per un altro valore qualunque $l_{1}$ di $x$ pel quale $X_{\psi 1}, \%$ fosse nullo, si perverrebbe ad un risultato analogo; da ciò è facile concludere che esistono $m^{n-1}$ funzioni intere della $x$

$$
\lambda_{0}, \quad \lambda_{1}, \ldots \lambda_{m^{n-1}}
$$

delle quali pero alcune possono essere anche eguali a costanti diverse da zero, tali che posto

$$
X_{\varphi, \%}=\lambda_{\varphi} T_{\varphi, \%}
$$

nessuna delle funzioni $T_{\varphi, \%}$ cosl definita sia nulla od infinita per valori finiti della $x$.

$$
V_{p}=\frac{\prod_{q=0}^{q=m^{n-1}-1} \prod_{p=0}^{p=m-1} X_{q, p}}{\prod_{p=0} \prod_{p=0} X_{\varphi, p}},
$$

ed osservando che per la (11) si ha

$$
1+\alpha^{m^{n-1}}+\alpha^{2 m^{n-1}}+\cdots+\alpha^{(m-1) m^{n-1}}=0
$$

poichè $\alpha$ è radice primitiva della (3), e quindi

$$
\begin{aligned}
& \int \frac{f d x}{\frac{1}{m^{n}}}=\frac{A}{m} \sum_{q=0}^{q=m^{n-1}-1} \sum_{p=0}^{p=n+1} \log \left(X_{q, p}^{m}\right)^{q+m^{n-1} p}+\frac{A}{m} \sum_{q=0}^{q=m^{n-1}-1} \sum_{p=0}^{p=m-1} V_{q}^{q+\alpha^{q-1} p p} \\
& =\frac{A}{m} \sum_{q=0}^{q=n^{n-1}-1} \sum_{p=0}^{p=m-1} \log \left(X_{q, p}^{n t} V_{q}\right)^{q+1 m^{n-1} p}
\end{aligned}
$$

Ma $X_{q, \chi}^{m} V_{\varphi}$ è della forma attribuita a $X_{\varphi, y}$ dalla $(4)$ e si passa da $X_{0,0}^{\text {m }} V_{0}$ a $X_{\rho, \gamma}^{m} V_{\rho}$ col sostituire nella prima di queste due funzioni $\alpha^{\varphi+m^{n-1} \psi} \theta^{\frac{1}{m^{n}}}$ al $\operatorname{lnog} 0$ di $0^{\frac{1}{m^{n}}}$, sostituzione colla quale si passa da $X_{0,0}$ a $X_{p, \chi}$, e facendo

$$
U_{p, ?}=X_{\varphi, \psi}^{m} V_{\varphi, 7} \text { e } \xi=\lambda_{0}^{m} \lambda_{1}^{n n} \ldots \lambda_{m^{n-1}-1}^{m}
$$


poichè

si ha

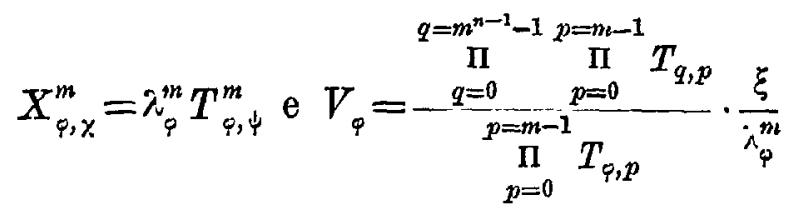

ponendo

$$
U_{\varphi, \chi}=X_{\varphi, \chi}^{m} V_{\varphi}=\xi T_{\varphi, \chi}^{m} \frac{\prod_{q=0} \prod_{p=0}^{q=m^{n-1}-1} T_{q, p}^{p=n-1}}{\prod_{p=0}^{p=m-1} T_{\varphi, p}}=\xi R_{\varphi, \%,}
$$

$$
R_{\varphi, \chi}=T_{\varphi, \chi}^{m} \frac{\prod_{q=0}^{q=m^{n-1}-1} \prod_{p=0}^{p=m-1} T_{q, p}}{\prod_{p=0} \prod_{p=0}}
$$

per cui $R_{\rho, \%}$ è differente da zero ed è finito per valori finiti della $x$. La (15) diviene dunque

$$
\int \frac{f d x}{\theta^{\frac{1}{m^{n}}}}=\frac{A}{m} \sum_{q=0}^{q=m^{n-1}-1} \sum_{p=0}^{p=m-1} \log \left(X_{q, p}^{n} V_{q}\right)^{\alpha^{q+m^{n-1} p}}=\frac{A}{m} \sum_{q=0}^{q=m^{n-1}-1} \sum_{p=0}^{p=m-1} U_{q, p}^{\alpha+m^{n-1} p}
$$

ma $\frac{A}{m}$ è una costante e $U_{\varphi, \%}$ è della forma, assegnata per $X_{\%, \%}$ dalla (4); dunque scrivendo nell' ultimo membro della (17) $A$ al luogo di $\frac{A}{m}$ e $X_{\varphi, \%}$ al luogo di $U_{\dot{c}, \%}$ otteremo una relazione della forma della (5) soltanto, che in essa, ci sarà lecito supporre che le $X_{p, x}$ soddisfino alla

$$
X_{\varphi, \%}=\xi R_{\dot{\epsilon}, \%}
$$

dove $R_{\varphi, \chi}$ rappresenta una funzione finita e diversa da zero per qualunque valore finito della $x$, e dove $\bar{\xi}$ è un polinomio intero rispetto alla stessa variabile.

4. Le (4) tenuto conto delle (18) ci forniscono

$$
\begin{aligned}
& \sum_{q=0}^{q=m n-1} \sum_{p=0}^{p=m-1} a^{-\omega\left(q+m^{n-1} p\right)} X_{q, p}=\xi^{q=m^{n-1}} \sum_{q=0}^{-1} \sum_{p=0}^{p=m-1} \alpha^{-\infty\left(q+m^{n-1} p\right)} R_{q, p} \\
& =\sum_{q=0}^{q=m^{n-1}-1} \sum_{p=0}^{p=m-1} \sum_{K=0}^{K=m^{n}-1} \alpha^{(K-\omega)\left(q+m^{n-1} p\right)} 0^{m^{n}} M_{K} \\
& =\sum_{K=0}^{K=n^{*}-1} \frac{\alpha^{(K-\omega) m^{n}}-1}{\alpha^{(K-\omega)}-1} M_{K} \theta^{\frac{K}{m^{n}}}
\end{aligned}
$$


ora, essendo w e $K$ interi e positivi ed entrambi minori di $m^{n}$, il numeratore di quest' ultima frazione sarà sempre zero, ed il denominatore differente da zero, eccetto il caso di $K=\omega$, nel qual caso la frazione ha per valore $m^{n}$, quindi finalmente avremo

$$
m^{n} M_{\omega} q^{\frac{\omega}{m^{n}}}=\xi^{q=m^{n-1}-1} \sum_{q=0}^{p=m-1} \sum_{p=0}^{-\omega\left(q+m^{n-1} p\right)} R_{q, p}=\bar{\xi} N_{\omega}
$$

ponendo

$$
N_{\omega}=\sum_{q=0}^{q=m^{n-1}-1} \sum_{p=0}^{p=m-1} \alpha^{-\omega\left(q+m^{\alpha-1} p\right)} R_{q, p}
$$

per cui evidentemente $N_{\omega}$ non diviene infinito per valori finiti della $x$, e facendo

dalla (19) si ricava

$$
\omega c_{b}=m^{n} r_{b, \omega}+s_{b, \omega}, \quad s \geqq 0 \text { e } s<m^{n},
$$

$$
N_{\omega}=m^{n} \frac{M_{\omega=0}^{b=g}\left(x-a_{b}\right)^{r, \omega}}{\xi} \cdot \prod_{b=0}^{b=g}\left(x-a_{b}\right)^{\frac{s_{b, \omega}}{m^{n}}}
$$

ed essendo $s_{b, \omega}<m^{n}$ e $N_{\omega}$ non divenendo infinito per valori finiti della $x$, dovrà lo stesso verificarsi pel quoziente

$$
P_{\omega}=\frac{M_{\omega}^{b=g} \mathrm{n}\left(x-a_{b}\right)^{r b, \omega}}{\xi}
$$

di due polinomi interi della $x$ e quindi dovrà ridursi ad un polinomio intero, da cui

$e$

$$
\begin{gathered}
N_{\omega}=m^{n} P_{\omega} \prod_{b=0}^{b=g}\left(x-a_{b}\right)^{\frac{s_{b, \omega}}{m^{*}}} \\
M_{\omega} \theta^{\frac{\omega}{m^{n}}}=\xi P_{\omega} \prod_{b=0}^{b=g}\left(x-a_{b}\right)^{\frac{s_{b, \alpha}}{m^{n}}}
\end{gathered}
$$

e le (18) divengono

$$
R_{\varphi, X}=\sum_{K=0}^{K=m^{n}-1} a^{-K\left(\varphi+m^{n-1} x\right)} P_{K} \prod_{b=0}^{b=g}\left(x-a_{b}\right)^{\frac{\delta_{b, K}}{m^{n}}} .
$$

E da qui si vede che $R_{\rho, x}$ ha la stessa forma di $X_{\varphi, x}$ soltanto che in esso al luogo di $\theta^{\frac{\Delta}{m^{n}}}=\prod_{b=0}^{b=g}\left(x-d_{b}\right)^{\frac{\omega c_{u}}{m^{n}}}$ si sostituisce $\prod_{b=0}^{b=g}\left(x-a_{b}\right)^{\frac{s_{b_{x} \omega}}{m^{n}}}$ dove $s_{b, \omega s}$ rappresenta il resto minimo positivo di $c_{b} \omega$ relativamente al modulo $m^{n}$. 
5. Se nella (5) sostituiamo $\xi R_{p, \%}$ al luogo di $X_{p, \%}$ ed osserviamo che

$$
1+\alpha^{3}+\alpha^{3}+\cdots \alpha^{m^{n}-2}+a^{m^{n}-1}=0
$$

si ricava

$$
\int \frac{f d x}{\theta^{\frac{1}{m^{n}}}}=A \sum_{q=0}^{q=m^{n-1-1}} \sum_{p=0}^{p=m-1} R_{q, p}^{\alpha^{q+m^{*-1} p}}
$$

deve $R_{p, x}$ conserva un valore finito e differente da zero per tutti i valori finiti della $x$. Quindi il prodotto

$$
\underset{q=0}{q=n^{n-1}-1} \underset{p=0}{p=m-1} R_{q, p}
$$

che è un polinomio intero rispetto alla $x$, e che non si annulla per valori finiti attribuiti a questa variabile, deve ridursi ad una costante. E l' equazione

$$
\prod_{q=0}^{q=m^{n-1}-1} \underset{n=0}{\prod} \prod_{n=0} R_{q, p}=\text { costante }
$$

è l' espressione del teorema dimostrato (pag. 21 volume suddetto) nella ipotesi di $n=1$ nell' indicata mia Nota esteso al caso generale di $n$ qualunque, per l'integrale

$$
\int \frac{f d x}{\theta^{\frac{1}{m^{n}}}}
$$

essendo $m$ un numero primo; con che ho conseguito l'oggetto del presente mio lavoro.

Sestri-Levante, 24 agosto 1874. 